11

\title{
Переходное структурное состояние порядок-порядок в монооксиде титана $\mathrm{TiO}_{1.0}$
}

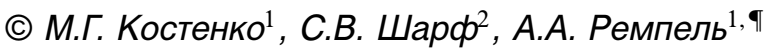 \\ ${ }^{1}$ Институт химии твердого тела УрО РАН, \\ Екатеринбург, Россия \\ ${ }^{2}$ Институт математики и механики УрО РАН, \\ Екатеринбург, Россия \\ ฯ E-mail: rempel@ihim.uran.ru
}

(Поступила в Редакцию 26 октября 2016 г.

В окончательной редакции 16 ноября 2016 г.)

\begin{abstract}
Предложен новый класс дефектных структур, в которых точечные дефекты кристаллической решетки занимают узлы одновременно двух разных сверхструктур. Образование данных структурных модификаций обусловлено равновесным фазовым переходом порядок-порядок второго рода, который не протекает до конца. На примере атомно-вакансионного упорядочения в монооксиде титана $\mathrm{TiO}_{1.0}$ исследовано допустимое соотношение между параметрами дальнего порядка в структурной модификации, образованной сочетанием моноклинной $\left(M_{5} X_{5}\right)_{\text {mon }}$ (пространственная группа $\left.C 2 / m(A 2 / m)\right)$ и кубической $\left(M_{5} X_{5}\right)_{\text {cub }}$ (пространственная группа $P(m 3 m)$ сверхструктур. С помощью термодинамических расчетов показано, что предлагаемая структурная модификация является равновесной и должна реализоваться вместо предполагаемой высокотемпературной кубической фазы $\left(\mathrm{Ti}_{5} \mathrm{O}_{5}\right)_{\text {cub. }}$.
\end{abstract}

Работа выполнена при финансовой поддержке РФФИ (проект № 14-02-00636).

Вычисления проведены на суперкомпьютере „Уран“ ИММ УрО РАН.

DOI: 10.21883/FTT.2017.06.44489.400

Для многих соединений переходных металлов характерно высокое содержание структурных вакансий [1]. Структурная вакансия представляет собой узел кристаллической решетки, не занятый атомом. Соединения со структурными вакансиями в настоящее время активно исследуются в связи с уникальностью их физикохимических свойств [2-8]. В зависимости от количества и способа размещения вакансий по узлам базисной кристаллической структуры возможно образование множества разнообразных фаз и модификаций [1]. В то же время особенности дефектных кристаллических структур с вакансиями, а также их связь с физическими свойствами до сих пор полностью не объяснены. Показательным примером является нестехиометрический монооксид титана $\mathrm{TiO}_{y}$ [9-24]. Соединение обладает базисной структурой $B 1$ и содержит структурные вакансии как в металлической, так и в неметаллической подрешетке. Доля вакантных узлов при стехиометрическом составе $(y=1)$ около 15\% для каждой подрешетки. При отклонении от стехиометрии содержание вакансий в одной из подрешеток может достигать $25 \mathrm{at} \%$ [12]. С учетом дефектности реальный состав соединения записывают в виде $\mathrm{TiO}_{y} \equiv \mathrm{Ti}_{x} \mathrm{O}_{z}$ или $M_{x} \mathbf{\square}_{1-x} X_{z \square 1-z}$, где $y=z / x$, 口 и - структурные вакансии металлической (титановой) и неметаллической (кислородной) подрешеток соответственно.

Упорядочение вакансий в монооксиде титана обнаружено авторами $[9,10]$, а также $[11]$. В работах $[9,10]$ подробно исследована упорядоченная моноклинная фаза со сверхструктурой $\left(M_{5} X_{5}\right)_{\text {mon }}$ (пространственная группа
$A 2 / m(c 2 / m))$, которая образуется из неупорядоченной кубической фазы в результате отжига в течение $50 \mathrm{~h}$ при температуре $950^{\circ} \mathrm{C}$. При обработке данных эксперимента по электронной микродифракции предложена модель распределения вакансий в упорядоченной фазе (рис. $1, a)$ и найдены параметры элементарной ячейки. В работе [11] помимо моноклинной фазы, термодинамически равновесной ниже $980^{\circ} \mathrm{C}$, упоминается фаза с кубической симметрией, пространственную группу которой точно определить не удалось. Упорядоченная кубическая фаза равновесна в диапазоне температур от 980 до $1250^{\circ} \mathrm{C}$, выше $1250^{\circ} \mathrm{C}$ равновесна неупорядоченная кубическая фаза, симметрия которой соответствует симметрии базисной кристаллической структуры $B 1$.

Позднее эксперименты по упорядочению структурных вакансий проводились авторами $[13,14]$. Образцы неупорядоченной кубической фазы отжигались при температуре $1057^{\circ} \mathrm{C}$ в течение $3 \mathrm{~h}$, а затем их структура исследовались методом рентгеновской дифракции. На рентгенограмме $[13,14]$ отожженных образцов обнаружены сверхструктурные рефлексы, анализ которых привел к той же модели моноклинной фазы, которая была предложена в работах $[9,10]$. Однако теоретическая рентгенограмма, рассчитанная для сверхструктуры $\left(M_{5} X_{5}\right)_{\mathrm{mon}}$, не дала удовлетворительного согласия с экспериментальной. Образцы упорядоченного монооксида титана, синтезированные авторами $[9,10]$, в дальнейшем исследовались методом электронной микродифракции [15-17]. Результаты [15-17] также не удается исчерпывающе объяснить с помощью модели моноклинной сверхструктуры 


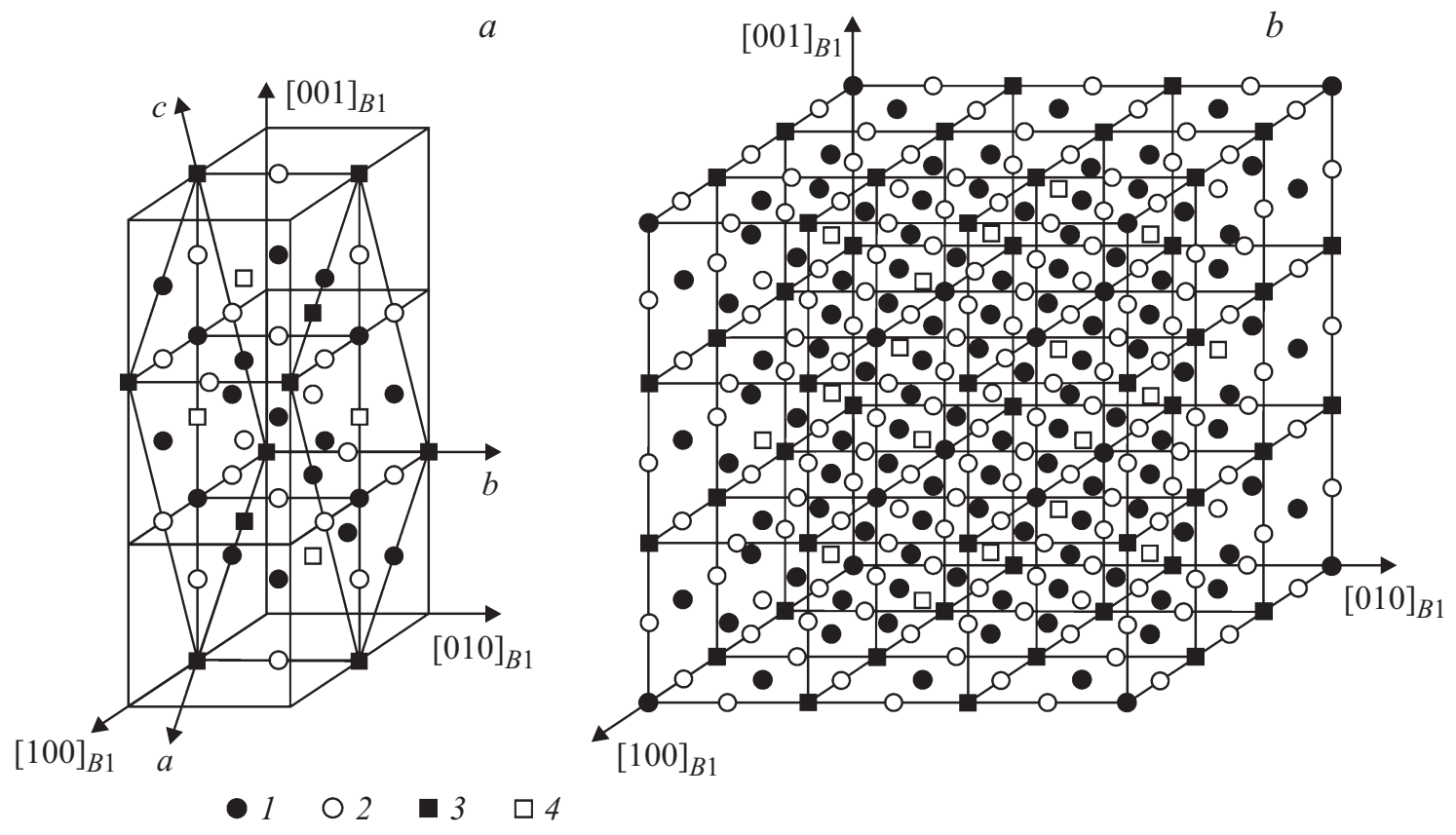

Рис. 1. Положение моноклинной $\left(M_{5} X_{5}\right)_{\text {mon }}[13,14](a)$ и кубической $\left(M_{5} X_{5}\right)_{\text {cub }}[18-20](b)$ элементарных ячеек в базисной структуре $B 1.1$ - позиция подрешетки атомов титана, 2 - позиция подрешетки атомов кислорода, 3 - позиции подрешетки титановых вакансий, 4 - позиции подрешетки кислородных вакансий. Показаны кристаллографические направления структуры $B 1$ и моноклинной сверхструктуры.

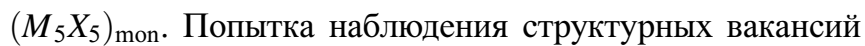
в направлении $[112]_{B 1}$ методом просвечивающей электронной микроскопии с высоким атомным разрешением, предпринятая в [15,16], не дала периодическую картину расположения вакансионных каналов, свойственных моноклинной сверхструктуре.

Одно из возможных объяснений противоречий в результатах [13-17] дано в работах [18-20], в которых предложена модель кубической сверхструктуры $\left(M_{5} X_{5}\right)_{\text {cub }}$ (пространственная группа $P m \overline{3} m$ ), вероятно наблюдаемой в эксперименте [10] (рис. 1, $b$ ). Данная структурная модель удовлетворительно описывает распределение сверхструктурных рефлексов на картине микродифракции [15-17], но не позволяет дать исчерпывающее объяснение результатам [13,14].

Причина разногласий [13-20], по-видимому, заключается в том, что авторами [13-20] рассматривался случай идеального дальнего порядка в сверхструктуpax, когда все вакансии располагаются строго в узлах образующейся при упорядочении подрешетки вакансий. В реальных упорядоченных фазах могут наблюдаться эффекты, связанные с частичным разупорядочением, при котором часть вакансий остается в подрешетке атомов. В частности, в работе [24] обнаружено понижение энергии основного состояния соединения при введении в структуру частично упорядоченной модификации корреляционного ближнего порядка $[1,25]$ одновременно с дальним порядком.

В настоящей работе предложено и исследовано принципиально новое структурное состояние, при котором остающиеся в подрешетке атомов вакансии размещаются не по типу корреляционного ближнего порядка $[1,25]$, а таким образом, что они занимают узлы вакансионной подрешетки другой сверхструктуры. В этом случае в упорядоченной фазе сосуществуют одновременно две разные сверхструктуры, а образованную таким образом структурную модификацию можно рассматривать как переходное состояние между двумя упорядоченными фазами.

Рассмотрим особенности структуры переходного состояния на примере сочетания сверхструктур $\left(M_{5} X_{5}\right)_{\text {mon }} \equiv\left(M_{10 \square} X_{10 \square 2}\right)_{\text {mon }} \quad$ и $\quad\left(M_{5} X_{5}\right)_{\text {cub }} \equiv$ $\equiv\left(M_{90 \square}{ }_{18} X_{90 \square 18}\right)_{\text {cub }} \quad$ (в обозначении сверхструктур с учетом состава указано количество вакансий в их элементарных ячейках [18]). При пространственном совмещении двух сверхструктур часть узлов подрешеток вакансий будет принадлежать только моноклинной сверхструктуре, часть - только кубической, а часть окажется общей. Степень упорядоченности вакансий в сверхструктуре характеризуется параметром дальнего порядка $\eta$, который, согласно $[13,14,18-20]$, связан с количеством вакансий $v$ в подрешетке вакансий сверхструктур $\left(M_{5} X_{5}\right)_{\text {mon }}$ и $\left(M_{5} X_{5}\right)_{\text {cub }}$ соотношением

$$
\eta=\frac{6}{5} v-\frac{1}{5}
$$

Предлагаемую переходную структуру следует описывать двумя параметрами дальнего порядка: вычисленным для моноклинной структуры $\eta_{\text {mon }}$ и вычисленным для кубической структуры $\eta_{\text {cub. }}$ Рассмотрим, какими 
могут быть соотношения между этими параметрами.

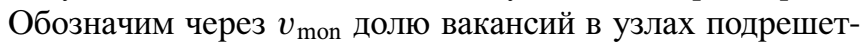
ки вакансий моноклинной сверхструктуры, которые не являются общими с узлами вакансионной подрешетки кубической сверхструктуры, соответствующую долю в кубической сверхструктуре - через $v_{\text {cub }}$, а долю вакансий в общих для двух сверхструктур узлах вакансионных подрешеток - через $v_{\text {joint. }}$ Суммарная доля вакансий в подрешетке вакансий моноклинной сверхструктуры составит $v_{\text {mon }}+v_{\text {joint. }}$ Если вакансии располагаются только в узлах подрешеток вакансий, то справедливо соотношение $v_{\text {mon }}+v_{\text {cub }}+v_{\text {joint }}=1$, с учетом которого из выражения (1), записанного отдельно для моноклинной и кубической сверхструктур, можно найти, что

$$
\eta_{\text {cub }}=-\eta_{\text {mon }}+\frac{6}{5} v_{\text {joint }}+\frac{4}{5} \text {. }
$$

Таким образом, область допустимых соотношений между параметрами дальнего порядка будет определяться вертикальным сдвигом прямой, описываемой уравнением (2), а максимальная величина сдвига будет зависеть только от количества общих для двух сверхструктур узлов подрешеток вакансий.

При анализе вариантов возможного пространственного размещения одной сверхструктуры относительно другой в матрице базисной кристаллической структуры $B 1$ обнаружено, что количество общих узлов подрешетки вакансий может составлять $0,22.23$ или $38.89 \%$ от общего количества вакансий. Соответственно существует три варианта изменения $v_{\text {joint }}: v_{\text {joint }}=0,0 \leq v_{\text {joint }} \leq 0.2223$ и $0 \leq v_{\text {joint }} \leq 0.3889$. В первом случае область допустимых значений вырождается в линию. В двух других случаях область допустимых значений помимо выражения (1) будет определяться условиями $\eta_{\mathrm{mon}} \geq \eta_{\operatorname{mon}}^{\min }$ и $\eta_{\mathrm{cub}} \geq \eta_{\mathrm{cub}}^{\mathrm{min}}$, поскольку в противном случае чисто моноклинных или чисто кубических вакансионных позиций будет недостаточно, чтобы вместить все имеющееся в соединении количество вакансий. Значения $\eta_{\operatorname{mon}}^{\min }$ и $\eta_{\text {cub }}^{\min }$ находятся из выражения (1) подстановкой $v=0.2223$ или $v=0.3889$ и составляют $\eta_{\mathrm{mon}}^{\min }=\eta_{\text {cub }}^{\min }=0.067$ и $\eta_{\mathrm{mon}}^{\min }=\eta_{\mathrm{cub}}^{\min }=0.2667$ соответственно.

На рис. 2 изображена область допустимых значений параметров для случая $0 \leq v_{\text {joint }} \leq 0.3889$. В точке $A(0.2667,1.0)$ все чисто моноклинные позиции подрешетки вакансий заняты атомами, в точке $B(1.0,0.2667)$ наблюдается аналогичная ситуация для кубической сверхструктуры. Точки, лежащие на отрезке $C D$, характеризуют структурные состояния, при которых количество вакансий в общих вакансионных позициях равно нулю. При движении по отрезку $B E$ вакансии из чисто моноклинных и общих позиций равновероятно переходят в чисто кубические вакансионные позиции. Формально структурный переход моноклинной фазы в кубическую можно моделировать движением по любой произвольной траектории внутри области $A B C D$. Обоснованным выглядит движение по отрезку $B A$, поскольку в этом случае требуется наименьшее количество перемещений вакансий из одной сверхструктуры в другую.

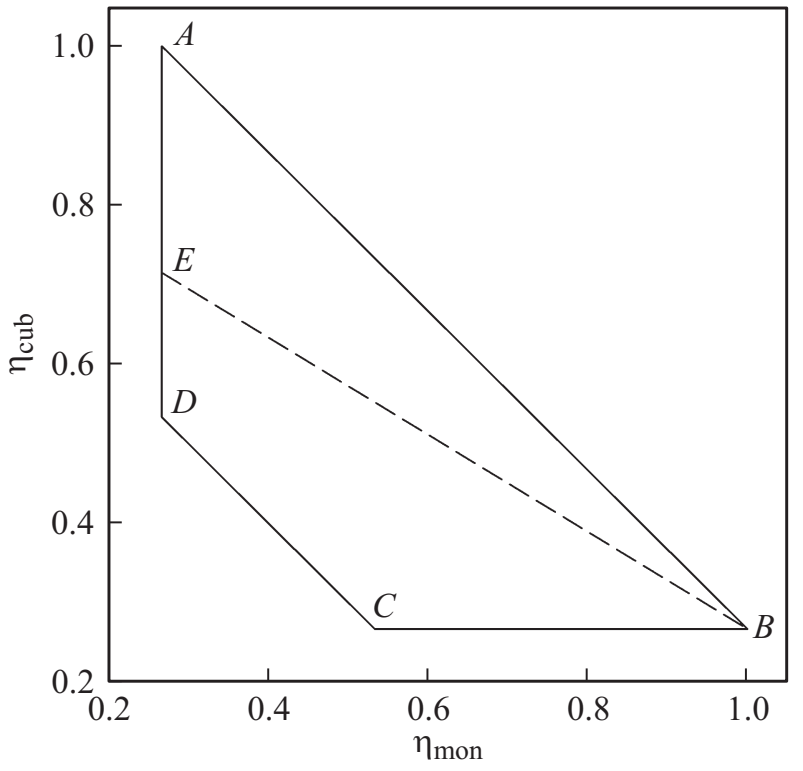

Рис. 2. Область возможных соотношений между параметрами дальнего порядка моноклинной и кубической сверхструктур $M_{5} X_{5}$ для случая $0 \leq v_{\text {joint }} \leq 0.3889$.

Рассмотрим термодинамическое обоснование существования переходного состояния $\left(M_{5} X_{5}\right)_{\text {mon }}-\left(M_{5} X_{5}\right)_{\text {cub }}$ в монооксиде титана. Равновесная структурная модификация соответствует минимуму свободной энергии $F=E-T S$ при данной температуре $T$. Вычисление точных зависимостей внутренней энергии $E$ и энтропии $S$ от параметров порядка $a b$ inito методами в настоящее время не представляется возможным. Тем не менее возможна качественная оценка поведения системы при различных температурах, а также определение рода фазового перехода с использованием простых моделей [26,27]. В монооксиде титана существенным является ионный тип связи [21-23], поэтому при расчете внутренней энергии возможно использование модели точечных зарядов. Несмотря на то что данная модель не позволяет рассчитать точные значения критических параметров порядка и температур фазовых переходов, она приводит к верным выводам о качественном поведении системы с повышением температуры [26]. Корректное использование более точных методов для расчета энергии $E$ [27] затруднительно из-за слишком большого размера элементарной ячейки кубической сверхструктуры. Будем считать, что вместо атомов в узлах решетки размещаются точечные заряды $+2 e$ (титан) и $-2 e$ (кислород). Заряд вакансий примем равным нулю. Энергия взаимодействия $\varepsilon_{i j}$ двух точечных зарядов $q_{i}$ и $q_{j}$, отдаленных на расстояние $r_{i j}$, определяется формулой

$$
\varepsilon_{i j}=\frac{k}{r_{i j}} q_{i} q_{j},
$$

где $k$ - постоянная. Чтобы найти полную энергию $E$ электростатического взаимодействия ионов кристалла, 

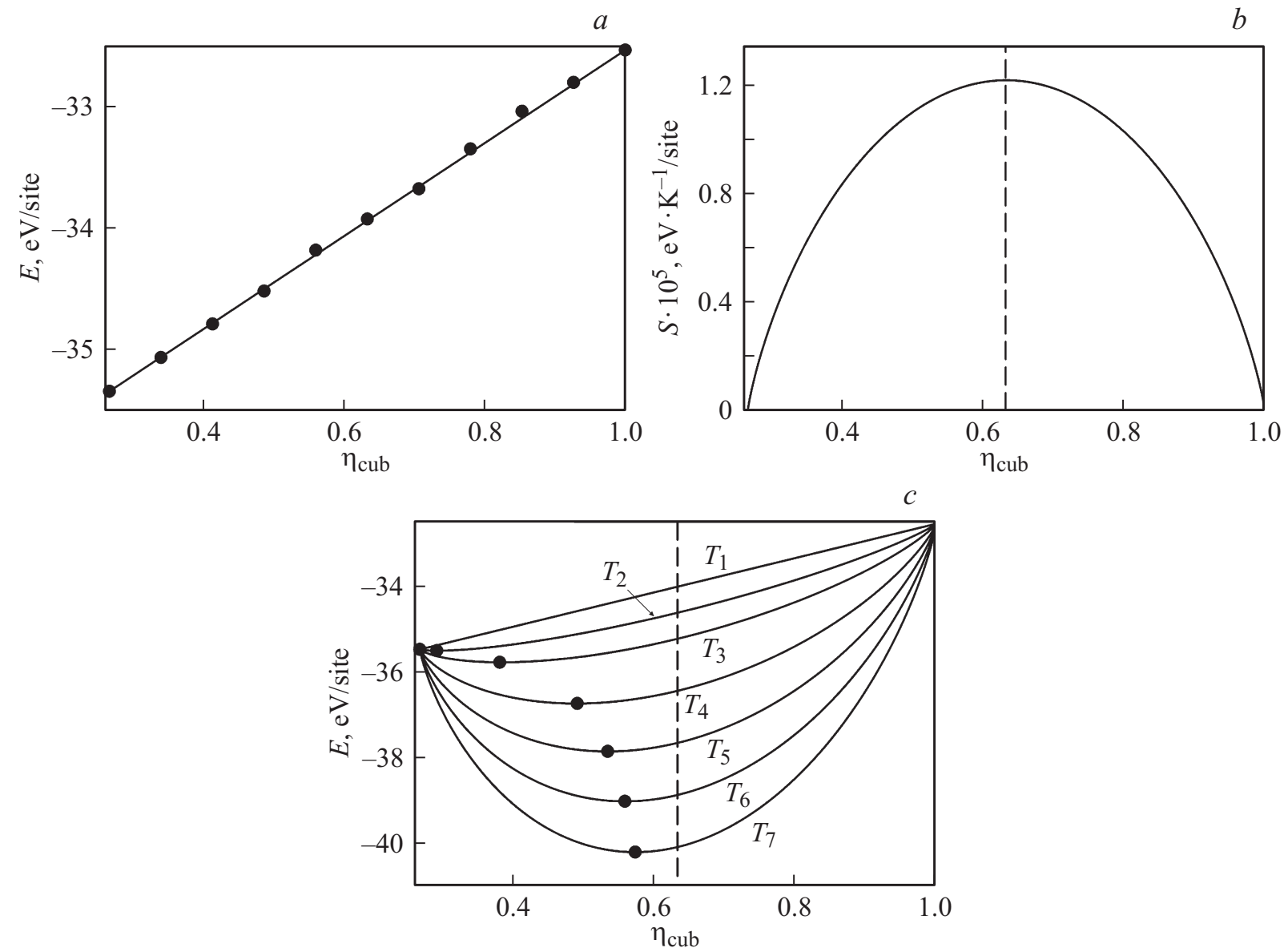

Рис. 3. Зависимости внутренней энергии $(a)$, конфигурационной энтропии $(b)$ и свободной энергии при различных температурах $\left(T_{1}<T_{2}<T_{3}<T_{4}<T_{5}<T_{6}<T_{7}\right)(c)$ от параметра дальнего порядка кубической сверхструктуры $\left(M_{5} X_{5}\right)_{\text {cub. }}$ Отмечены минимумы свободной энергии. Штриховой линией обозначена асимптота для минимумов, соответствующая максимуму энтропии.

нужно суммировать энергию взаимодействия всех возможных пар ионов $i, j$

$$
E=\frac{1}{2} \sum_{i=1}^{N} \sum_{\substack{j=1 \\ i \neq j}}^{N} \varepsilon_{i j},
$$

где $N$ - суммарное количество узлов решетки $B 1$ кристалла. Зависимость $E\left(\eta_{\text {cub }}\right)$, рассчитанная с помощью суперкомпьютерного моделирования для кристалла кубической формы размером $204 \times 204 \times 204$ узлов при переходе из моноклинной структуры в кубическую по траектории $B A$, представлена на рис. 3, $a$. Данные при $\eta_{\text {cub }} \neq 0.2667\left(\eta_{\text {mon }} \neq 1\right)$ и $\eta_{\text {cub }} \neq 1\left(\eta_{\text {mon }} \neq 0.2667\right)$ получены усреднением для двадцати кристаллов с различным конкретным расположением вакансий в чисто моноклинных и чисто кубических вакансионных позициях. Результаты вычислений аппроксимированы уравнением прямой

$$
E=-36.366+3.836 \eta_{\text {cub }}[\mathrm{eV} / \mathrm{site}]
$$

Энтропия может быть оценена как конфигурационная энтропия, возникающая вследствие некоторой доли беспорядка в чисто моноклинных и чисто кубических вакансионных позициях при $\eta_{\text {cub }} \neq 0.2667$ и $\eta_{\text {cub }} \neq 1$, и найдена по формуле Больцмана

$$
S=k_{\mathrm{B}} \ln \Omega,
$$

где $\Omega$ - число микросостояний системы, с помощью которых реализуется данное макросостояние, $k_{\mathrm{B}}-$ постоянная Больцмана. Величина $\Omega$ определяется для некоторой группы узлов комбинаторной формулой

$$
\Omega=\frac{M !}{k !(M-K) !}
$$

где $M$ - число узлов в рассматриваемой группе, $K$ число узлов данной подрешетки, занятых атомами либо вакансиями. В принятой для расчета модели беспорядок возможен только в чисто моноклинных или чисто кубических вакансионных позициях. Расчет зависимости $E\left(\eta_{\text {cub }}\right)$ следует вести отдельно по двум группам позиций, а энтропия кристалла определяется как сумма энтропий рассматриваемых групп

$$
S_{\mathrm{tot}}=S_{\mathrm{mon}}+S_{\mathrm{cub}}
$$


Логарифмирование факториалов осуществляется по формуле Стирлинга

$$
\ln N ! \approx N \ln N-N
$$

Тогда с учетом свойств логарифма выражение (3) преобразуется к виду

$$
S=k_{\mathrm{B}}(M \ln K-K \ln K-(M-K) \ln (M-K)),
$$

и задача, таким образом, сводится к нахождению величин $M$ и $K$.

Если процессы упорядочения в титановой и кислородной подрешетках происходят одинаковым образом, то $M$ и $K$ можно вычислять суммарно для металлических и кислородных вакансионных позиций. Если всего в кристалле $N$ узлов структуры $B 1$, то, согласно особенностям сверхструктур $M_{5} X_{5}$, на долю вакансий приходится $\frac{1}{6} N$ узлов, причем в принятой модели $38.89 \%$ из них не вносят вклада в конфигурационную энтропию. Поэтому $M$ составит

$$
M=\frac{1}{6} 0.6111 N .
$$

$K$ в отличие от $M$ является зависящей от параметра порядка величиной. Согласно [6-13] доля атомов в подрешетке вакансий сверхструктуры типа $M_{5} X_{5}$ составляет $\frac{5}{6}-\frac{5}{6} \eta$. При этом, поскольку $38.89 \%$ узлов всегда вакантно, количество атомов, вносящих вклад в энтропию, будет больше в $1 / 0.6111$ раз. В итоге получается, что

$$
K=M\left(\frac{5}{6}-\frac{5}{6} \eta\right) / 0.6111 .
$$

Для удобства вместо $K$ введем величину $K^{\prime}=K / M$, а вместо $M-$ величину $M^{\prime}=M / N$. С учетом (6) и (7) выражение (5) преобразуется к виду

$$
S=k_{\mathrm{B}} N M^{\prime}\left(\ln M^{\prime}-K^{\prime} \ln M^{\prime} K^{\prime}-\left(1-K^{\prime}\right) \ln \left(M^{\prime}-M^{\prime} K^{\prime}\right)\right) .
$$

Зависимость $S_{\text {tot }}\left(\eta_{\text {cub }}\right)$, вычисленная с учетом (4), (8) и соотношения (2) между $\eta_{\text {cub }}$ и $\eta_{\text {mon }}$ при $v_{\text {joint }}=0.3889$, приведена на рис. 3, $b$. Конфигурационная энтропия имеет максимум при $\eta_{\text {cub }}=0.6333$, т. е. когда вакансии поровну распределены между чисто моноклинными и чисто кубическими вакансионными позициями.

На рис. 3, $c$ показаны зависимости свободной энергии $F=E-T S$ от параметра дальнего порядка кубической сверхструктуры, вычисленные при различных температурах. Равновесные значения параметра дальнего порядка, соответствующие минимуму свободной энергии, постепенно увеличиваются с ростом температуры и асимптотически приближаются к значению 0.6333. Подобное поведение структурного параметра характерно для фазового перехода второго рода. В действительности максимально возможное значение параметра порядка не будет достигнуто, так как при некоторой температуре выгодным окажется состояние с еще большей конфигурационной энтропией, вызванное разупорядочением вакансий и в кубической, и в моноклинной сверхструктурах. Произойдет фазовый переход порядок-беспорядок с образованием неупорядоченной кубической фазы. Необходимо отметить, что превращение порядок-порядок не протекает до конца, т.е. идеально упорядоченная кубическая фаза $\left(\mathrm{Ti}_{5} \mathrm{O}_{5}\right)_{\text {cub }}[11,18-20]$ ни при каких температурах не является термодинамически равновесной, вместо нее образуется особое структурное состояние, в котором вакансии распределены по подрешеткам сразу двух сверхструктур. Последовательность равновесных фазовых превращений, происходящих в монооксиде титана при повышении температуры, должна выглядеть следующим образом: моноклинная фаза $\left(\mathrm{Ti}_{5} \mathrm{O}_{5}\right)_{\text {mon }}-$ переходное состояние $\left(\mathrm{Ti}_{5} \mathrm{O}_{5}\right)_{\text {mon }}-\left(\mathrm{Ti}_{5} \mathrm{O}_{5}\right)_{\text {cub }}$-неупорядоченная кубическая фаза $\mathrm{TiO}_{1.0}$. В эксперименте $[13,14]$ низкотемпературную моноклинную упорядоченную фазу не удалось получить, так как использовались более высокие температуры и меньшее время отжига, чем в оригинальных работах $[9,10]$.

Таким образом, в настоящей работе предложена новая модель распределения структурных вакансий в упорядоченных фазах. Эта модель принципиально отличается от предыдущих тем, что превращение из упорядоченного состояния в неупорядоченное проходит через частичное образование высокотемпературных сверхструктур, которые сами по себе не являются равновесными, а играют роль дополнительных и необходимы для увеличения энтропийного вклада в свободную энергию упорядоченной фазы при повышении температуры. Структурные особенности переходных состояний в соединениях с атомно-вакансионным упорядочением [1], а также их влияние на электронную структуру и физические свойства требует дальнейшего теоретического и экспериментального изучения. По аналогии со структурными вакансиями может быть рассмотрено перераспределение атомов замещения или внедрения. Интерес представляет исследование возможности образования переходных состояний в сплавах и твердых растворах, для которых известны низкотемпературные и высокотемпературные упорядоченные фазы. Многие высокотемпературные упорядоченные фазы, по-видимому, представляют собой сочетания двух или более сверхструктур, так как повышенные температуры предполагают некоторую долю беспорядка вследствие роста вклада конфигурационной энтропии.

\section{Список литературы}

[1] A.I. Gusev, A.A. Rempel, A.J. Magerl. Disorder and order in strongly nonstoichiometric compounds. Transition metal carbides, nitrides and oxides. Springer, Berlin, etc. (2001). $607 \mathrm{p}$.

[2] V. Bragaglia, F. Arciprete, W. Zhang, A.M. mio, E. Zallo, K. Perumal, A. Giussani, S. Cecchi, J.E. Boschker, H. Riechert, S. Privitera, E. Rimini, R. Mazzarello, R. Calargo. Sci. Rep. 6, 23843 (2016).

[3] C. Xie, A.R. Oganov, D. Li, T.T. Debela, N. Liu, D. Dong, Q. Zeng. Phys. Chem. Chem. Phys. 18, 12299 (2016). 
[4] X. Chong, Y. Jiang, R. Zhou, J. Feng. RSC Adv. 4, 44959 (2014).

[5] А. И. Гусев. ЖЭТФ 147, 5, 984 (2015).

[6] А.А. Валеева, С.3. Назарова, А.А. Ремпель. Письма в ЖЭТФ 101, 4, 276 (2015).

[7] А.И. Гусев. ЖЭТФ 147, 1, 105 (2015).

[8] А.И. Гусев, А.С. Курлов, И.А. Бобриков, А.М. Балагуров. Письма в ЖЭТФ 102, 3, 179 (2015).

[9] D. Watanabe, J.R. Castles, A. Jostsons, A.S. Marlin. Nature 210, 934 (1966).

[10] D. Watanabe, J.R. Castles, A. Jostsons, A.S. Marlin. Acta Cryst. 23, 307 (1967).

[11] E. Hilti. Naturwissenschaften 55, 130 (1968).

[12] M.D. Banus, T.B. Reed, A.J. Strauss. Phys. Rev. B 5, 2775 (1972).

[13] А.А. Валеева, А.А. Ремпель, А.И. Гусев. Письма в ЖЭТФ 71, 11, 675 (2000)

[14] А.А. Валеева, А.А. Ремпель, А.И. Гусев. Неорган. материалы 37, 716 (2001).

[15] А.А. Валеева, Г. Танг, А.И. Гусев, А.А. Ремпель. Письма в ЖЭТФ 77, 1, 28 (2003).

[16] А.А. Валеева, Г. Танг, А.И. Гусев, А.А. Ремпель. ФТТ 45, 1, 84 (2003).

[17] А.А. Валеева, А.И. Гусев. ФТТ 48, 9, 1598 (2006).

[18] А.И. Гусев, А.А. Валеева. Письма в ЖЭТФ 96, 6, 400 (2012).

[19] А.И. Гусев. ЖЭТФ 144, 2, 340 (2013).

[20] A.I. Gusev. J. Solid State Chem. 199, 181 (2013).

[21] J.B. Goodenough. Phys. Rev. B 5, 2764 (1972).

[22] J. Graciani, A. Márquez, J.F. Sanz. Phys. Rev. B 72, 054117 (2005).

[23] M.G. Kostenko, A.V. Lukoyanov, V.P. Zhukov, A.A. Rempel. J. Solid State Chem. 204, 146 (2013).

[24] М.Г. Костенко, А.А. Ремпель, С.В. Шарф, А.В. Лукоянов. Письма в ЖЭТФ 102, 2, 94 (2015).

[25] A.A. Rempel, A.I. Gusev. Phys. Status Solidi B 160, 389 (1990).

[26] М.Г. Костенко, А.А. Ремпель. ФТТ 53, 9, 1808 (2011).

[27] М.Г. Костенко, А.А. Ремпель, А.В. Лукоянов. ЖЭТФ 143, 6, 1097 (2013). 